\title{
Alterations in Brain and Immune Function Produced by Mindfulness Meditation
}

Richard J. Davidson, PhD, Jon Kabat-Zinn, PhD, Jessica Schumacher, MS, Melissa Rosenkranz, Ba, Daniel Muller, MD, PhD, Saki F. Santorelli, EdD, Ferris Urbanowski, MA, Anne Harrington, PhD, Katherine Bonus, MA, and John F. Sheridan, PhD

\begin{abstract}
Objective: The underlying changes in biological processes that are associated with reported changes in mental and physical health in response to meditation have not been systematically explored. We performed a randomized, controlled study on the effects on brain and immune function of a well-known and widely used 8-week clinical training program in mindfulness meditation applied in a work environment with healthy employees. Methods: We measured brain electrical activity before and immediately after, and then 4 months after an 8-week training program in mindfulness meditation. Twenty-five subjects were tested in the meditation group. A wait-list control group $(N=16)$ was tested at the same points in time as the meditators. At the end of the 8-week period, subjects in both groups were vaccinated with influenza vaccine. Results: We report for the first time significant increases in left-sided anterior activation, a pattern previously associated with positive affect, in the meditators compared with the nonmeditators. We also found significant increases in antibody titers to influenza vaccine among subjects in the meditation compared with those in the wait-list control group. Finally, the magnitude of increase in left-sided activation predicted the magnitude of antibody titer rise to the vaccine. Conclusions: These findings demonstrate that a short program in mindfulness meditation produces demonstrable effects on brain and immune function. These findings suggest that meditation may change brain and immune function in positive ways and underscore the need for additional research. Key words: meditation, mindfulness, EEG, immune function, brain asymmetry, influenza vaccine
\end{abstract}

HIV = human immunodeficiency virus; $\mathbf{N K}=$ natural killer cell; EEG = electroencephalography; EOG = electrooculography; PANAS $=$ Positive and Negative Affective Scale; MBSR = mindfulness-based stress reduction; MANOVA $=$ multivariate analysis of variance.

\section{INTRODUCTION}

W ith the widespread and growing use of meditative practices in hospitals and academic medical centers for outpatients presenting with a range of chronic stress and pain-related disorders and chronic diseases, under the umbrella of what has come to be called mind/body or integrative medicine, the question of possible biological mechanisms by which meditation may affect somatic, cognitive, and affective processes becomes increasingly important. Research on the biological concomitants of meditation practice is sparse and has mostly focused on changes that occur during a period of meditation compared with a resting control condition in a single experimental session (1-3). Whereas these studies have been informative, they tell us little about changes that are potentially more enduring. Moreover, virtually all forms of meditation profess to alter everyday behavior, effects that are by definition not restricted to the times during which formal

From Laboratory for Affective Neuroscience (R.J.D., J.S., M.R.), Department of Psychology, University of Wisconsin, Madison, Wisconsin; Stress Reduction Clinic, Division of Preventive and Behavioral Medicine (J.K.-Z., S.F.S., F.U.), Department of Medicine, University of Massachusetts Medical School, Worcester, Massachusetts; Departments of Medicine and Microbiology (D.M.), University of Wisconsin Medical School; Department of the History of Science (A.H.), Harvard University, Cambridge, Massachusetts; Departments of Preventive Cardiology and Sports Medicine (K.B.), University of Wisconsin-Madison Hospitals and Clinics Center for Mindfulness, Madison, Wisconsin; and Department of Oral Biology (J.F.S.), College of Dentistry, Ohio State University, Columbus, Ohio.

Address reprint requests to: Richard J. Davidson, PhD, Laboratory for Affective Neuroscience, University of Wisconsin, 1202 W. Johnson St., Madison, WI 53706. Email: rjdavids@facstaff.wisc.edu

Received for publication April 4, 2002; revision received December 27, 2002.

DOI: 10.1097/01.PSY.0000077505.67574.E3 meditation itself is practiced. Thus, in the current report, we focus not on the period of meditation itself, but rather on the more enduring changes that can be detected in baseline brain function as well as brain activity in response to specific emotional challenges.

We focus on emotion-related brain activity because meditation has been found in numerous studies to reduce anxiety and increase positive affect (4-8). In an extensive corpus of work on the functional neuroanatomical substrates of emotion and affective style, we have established that the frontal regions of the brain exhibit a specialization for certain forms of positive and negative emotion $(9,10)$. Left-sided activation in several anterior regions is observed during certain forms of positive emotion and in subjects with more dispositional positive affect $(10,11)$. We therefore hypothesized that because meditation decreases anxiety and increases positive affect, subjects who were practicing meditation should show increased left-sided activation in these territories compared with those in a wait-list control group.

Recent studies have established that greater relative leftsided anterior activation at baseline is associated with enhanced immune function using measures of NK activity (12, 13). There has been a paucity of serious research attention to possible immune alterations that might be produced by meditation (14). This is somewhat surprising in light of the fact that negative psychosocial influences on immunity have now been well established (15-17). Recent research indicates that relaxation and stress management procedures increase T-cytotoxic/suppressor $(\mathrm{CD} 3+\mathrm{CD})$ lymphocytes in HIV-infected men (18). On the basis of recent research demonstrating the negative impact of stressful life events on antibody titers in response to influenza vaccine (19), we vaccinated all subjects at the end of the 8-week meditation program (in mid November), along with the subjects in wait-list control group at the same time. We hypothesized that the meditators would show greater antibody titers in response to the vaccine compared with the subjects in the wait-list control group. On the basis of 
the association we have previously reported between anterior activation asymmetry and NK activity, we also predicted that the magnitude of change toward greater relative left-sided activation would be associated with a larger increase in antibody titers in response to the vaccine.

\section{METHODS}

Measures of brain electrical activity were recorded before random assignment to each of the two groups (Time 1) and then again immediately after (Time 2) and four months after (Time 3) the training period ended. Brain electrical activity, or EEG, and EOG (for correcting EEG for eye movements) was recorded during both baseline conditions and in response to a positive and negative emotion induction using methods that have been extensively described in previous research $(20,21)$. EEG was recorded from 27 sites distributed across the scalp and referenced to linked ears during 8 1-minute baseline trials, four with eyes open and four with eyes closed, presented in counterbalanced order according to our established procedures (22). EEG was also recorded during a 1-minute period before and a 3-minute period after subjects wrote about one of three of the most positive and negative experiences in their life. These events were listed on a questionnaire administered to subjects before the start of the entire protocol. For this task, the EEG was aggregated across the 1-minute period before and the 3-minute period after the writing itself. Data were not collected during writing because of movement artifact. The EEG was parsed into 1.024-second epochs, overlapped by $50 \%$ and then processed with the use of a fast Hartley transform method to derive measures of spectral power density in the $\alpha$-band $(8-13 \mathrm{~Hz})$, which is inversely related to activation $(20,22)$. Asymmetric activation was indexed using an asymmetry score that is computed by subtracting log-transformed left hemisphere $\alpha$-power densities from the comparable measure derived from homologous right-sided electrodes.

After each of the writing periods, subjects were given the PANAS (23) in state form. In addition, at each assessment, they were administered the PANAS in trait form, along with the Spielberger State-Trait Anxiety Inventory (24) in trait form. In addition, subjects in the meditation group were asked to provide daily reports of the frequency and number of minutes and techniques of formal meditation practice.

Blood draws were then obtained at 3 to 5 weeks and then again at 8 to 9 weeks after vaccination to examine antibody titers in response to the vaccine using the hemagglutination inhibition assay (19).

A total of 48 right-handed subjects who were employees of a biotechnology corporation in Madison, Wisconsin, were recruited to participate. Of these, 41 subjects completed some of the measures for at least two of the assessments. The initial laboratory evaluation was conducted before random group assignment. Subjects were then randomly assigned to the meditation group $(N=25 ; 19$ female $)$ and the wait-list control group $(N=16 ; 10$ female $)$ at a ratio of approximately $3: 2$. There were no differences between groups in the number of subjects who failed to complete the study. Average age of subjects was 36 years and did not differ between group (range $=23$ to 56 years). All but two subjects were white (one Asian-American in the treatment group; one South Asian Indian in the control group). Subjects in the wait-list control group were evaluated at each assessment period along with subjects in the meditation group. After completion of the last assessment, the wait-list control subjects were provided with an 8-week training program comparable to that provided to the subjects in the meditation group.

The meditation training (known as MBSR) was delivered by J.K.-Z., and was directly modeled on the MBSR intervention originally developed at the University of Massachusetts Medical Center $(25,26)$. The effects of MBSR have been reported in numerous clinical studies with diverse populations, as well as in medical students $(27,28)$. One study demonstrated significant effects of mindfulness on the rate of skin clearing in patients with moderate to severe psoriasis (29) Two recent reviews of MBSR research called for studies to elucidate potential mechanisms of action $(30,31)$.

The training consisted of a class that met weekly for 2.5 to 3 hours per class, along with a silent seven-hour retreat that was held during week 6 of the course. In addition, subjects were assigned home practice that consisted of formal and informal meditative practices that they were instructed to perform for 1 hour per day, 6 days per week, with the aid of guided audiotapes.

The statistical analysis of the data focused on the interactions between group (Meditation/Wait-list control) and time (Times 1-3, with the first assessment occurring before the intervention, Time 2 occurring immediately after the 8-week intervention and Time 3 occurring four months after the training period ended. MANOVAs were computed for each of the four anterior asymmetry measures. In addition to examining main effects and the interaction, linear trends were also tested. Follow-up ANOVAs on the separate time periods were performed.

\section{RESULTS}

\section{Affect and Anxiety Measures}

We evaluated self-report measures of positive and negative affect and anxiety before and after the training. There was a significant Group $\times$ Time interaction $[F(1,31)=5.45, p<$ $.05]$ on a measure of trait anxiety, the Spielberger State-Trait Anxiety Inventory (24), accounted for by a reduction in anxiety for subjects in the meditation group from Time 1 to Time $2[t(20)=2.86, p<.01$; see Figure 1$)$. There was no significant Group $\times$ Time interaction on the Positive and Negative Affect Scale (23). However, in light of the clear a priori predictions for the meditators to show significant decreases in negative affect with treatment, we tested change over time within each group. There was a significant decrease in trait negative affect with the mediators showing less negative affect at Times 2 and 3 compared with their negative affect at Time $1[t(20)=2.27$ and $t(21)=2.45$, respectively, $p<.05$ for both; not shown]. Subjects in the control group showed no change over time in negative affect $(t<1)$.

\section{Brain Electrical Activity Measures}

Based on previous findings linking asymmetric anterior activation to positive affect, we specifically examined changes in four anterior electrode sites (F3/4, FC7/8, T3/4, and $\mathrm{C} 3 / 4$ in the International 10/20 system) during both base-line periods and in response to the emotion inductions. We computed MANOVAs with Group and Time (Times 1-3) as factors and examined main effects and interactions, as well as linear

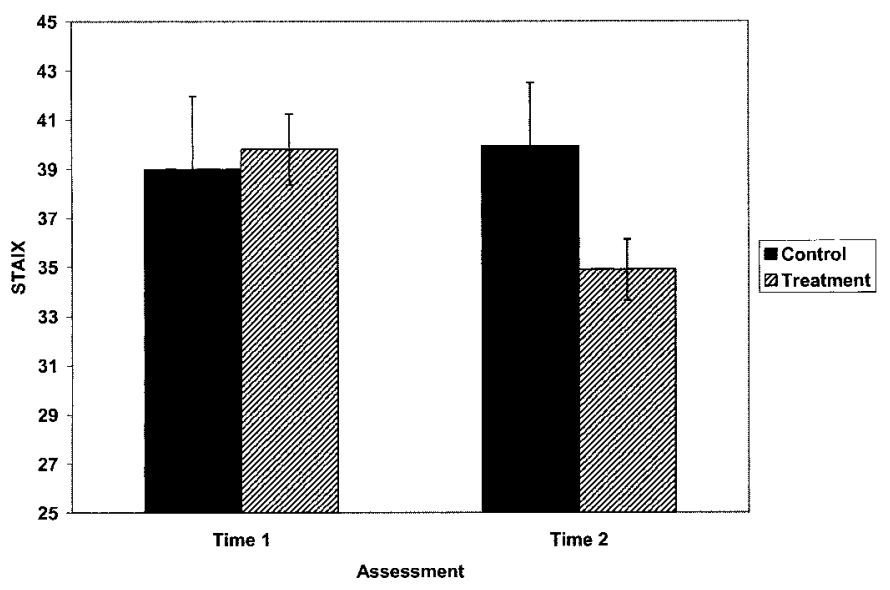

Fig. 1. Mean trait anxiety from the Spielberger State-Trait Anxiety Inventory (24) measured separately by group and time. Error bars reflect means $\pm \mathrm{SE}$. 
trends. For the baseline period assessments, there was a marginally significant Group $\times$ Time linear trend $(F(1,33)=$ $3.73, p=.06$ ) and a significant main effect for Group (across time periods; $F(1,33)=4.57, p=.04)$. When the comparison of change from baseline for each time period was examined, there was a significant Group $\times$ Time interaction $[F(1,37)=$ $5.14, p<.05]$ for the Time $1-3$ comparison and a marginally significant Group $\times$ Time interaction $[F(1,33)=2.82, p=$ .10] for the Time 1-Time 2 comparison for the central leads (C3/4). At Time 1, no group differences were present at baseline for any region. At both Time 2 and Time 3, meditators showed significantly greater relative left-sided activation at the central sites (C3/4) compared with the wait-list control group ( $p<.05$ for each; see Figure 2).

The omnibus MANOVA performed on the positive emotion induction condition revealed a marginally significant overall Group $\times$ Time interaction $[F(2,26)=2.52, p=.10]$ for the anterior temporal (T3/4) electrode leads. When the comparison of change from baseline for each time period was examined, there was a significant Group $\times$ Time interaction $[F(1,30)=4.82, p<.05]$ for the Time 1-Time 2 comparison. This same interaction for the Time 1-Time 3 comparison was marginally significant and in the same direction $[F(1,29)=$ $3.46, p=.07]$. In response to the positive emotion induction at Time 1 , no group differences were present in any region. However, meditators showed a significant increase in leftsided anterior temporal activation from Time 1 to Time 2 ( $p<$ $.05)$, whereas controls showed no change (Figure 3). There were no other significant Group $\times$ Time interactions for any other electrode site for the positive emotion induction.

In response to the negative affect induction, the omnibus MANOVA revealed a marginally significant linear trend for the Group $\times$ Time interaction $[F(1,27)=2.94, p<.10]$ for the anterior temporal leads. The Group $\times$ Time interaction for the Time 1-Time 2 comparison for the anterior temporal region (T3/4) was again in the same direction as the other interactions, but not significant $[F(1,31)=3.16$, $p=.08]$.

In response to the negative emotion induction for the central leads, an omnibus MANOVA revealed a marginally significant Group $\times$ Time interaction $[F(2,32)=2.78, p<.08]$, along with a marginally significant linear trend for this interaction $[F(1,33)=3.45, p=.07]$. In addition, there was a significant main effect for Group $[F(1,33)=6.78, p=.01]$. For the central leads, the Group $\times$ Time interaction for the Time 1 -Time 2 comparison was $F(1,33)=3.62, p=.07$, and for the Time 1-Time 3 comparison it was $F(1,37)=5.41, p<$ .05. Again, there were no group differences in any region at Time 1. At Times 2 and 3, subjects in the meditation group showed significantly greater left-sided activation (C3/C4) compared with subjects in the control group (for Time $2: p<$ .05 ; for Time $3: p<.01)$. The meditators evinced a significant increase in left-sided activation in this region from Time 1 to Time 2 ( $p<.05$; not shown) and Time $3(p<.05$ : Figure 4$)$.

There were no group differences present for any of the posterior electrodes sites for any of the conditions.

\section{Influenza Vaccine Antibody Titers}

In response to the influenza vaccine, the meditators displayed a significantly greater rise in antibody titers from the 4 to the 8 week blood draw compared with the controls $[t(33)=$ $2.05, p<.05$; Figure 5].

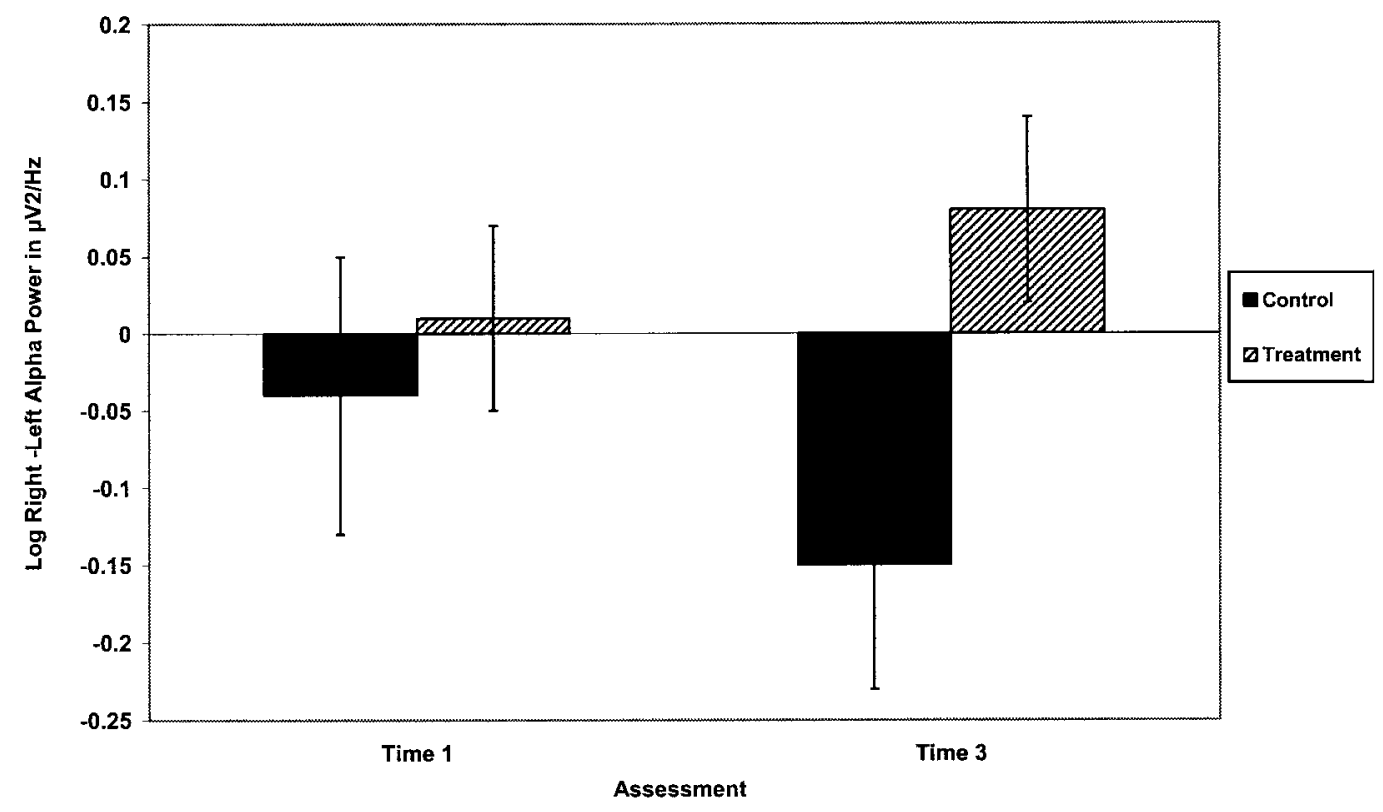

Fig. 2. Means $\pm \mathrm{SE}$ of asymmetric activation during baseline for subjects in the Meditation group and Control group during Time 1 (before random assignment, before treatment began) and Time 3. The ordinate is an asymmetric metric that represents right minus left log-transformed $\alpha$ power density from the $\mathrm{C} 4 / \mathrm{C} 3$ electrode sites. This is a standard index of asymmetric activation (20). Higher numbers on this indicate greater left-sided activation. 


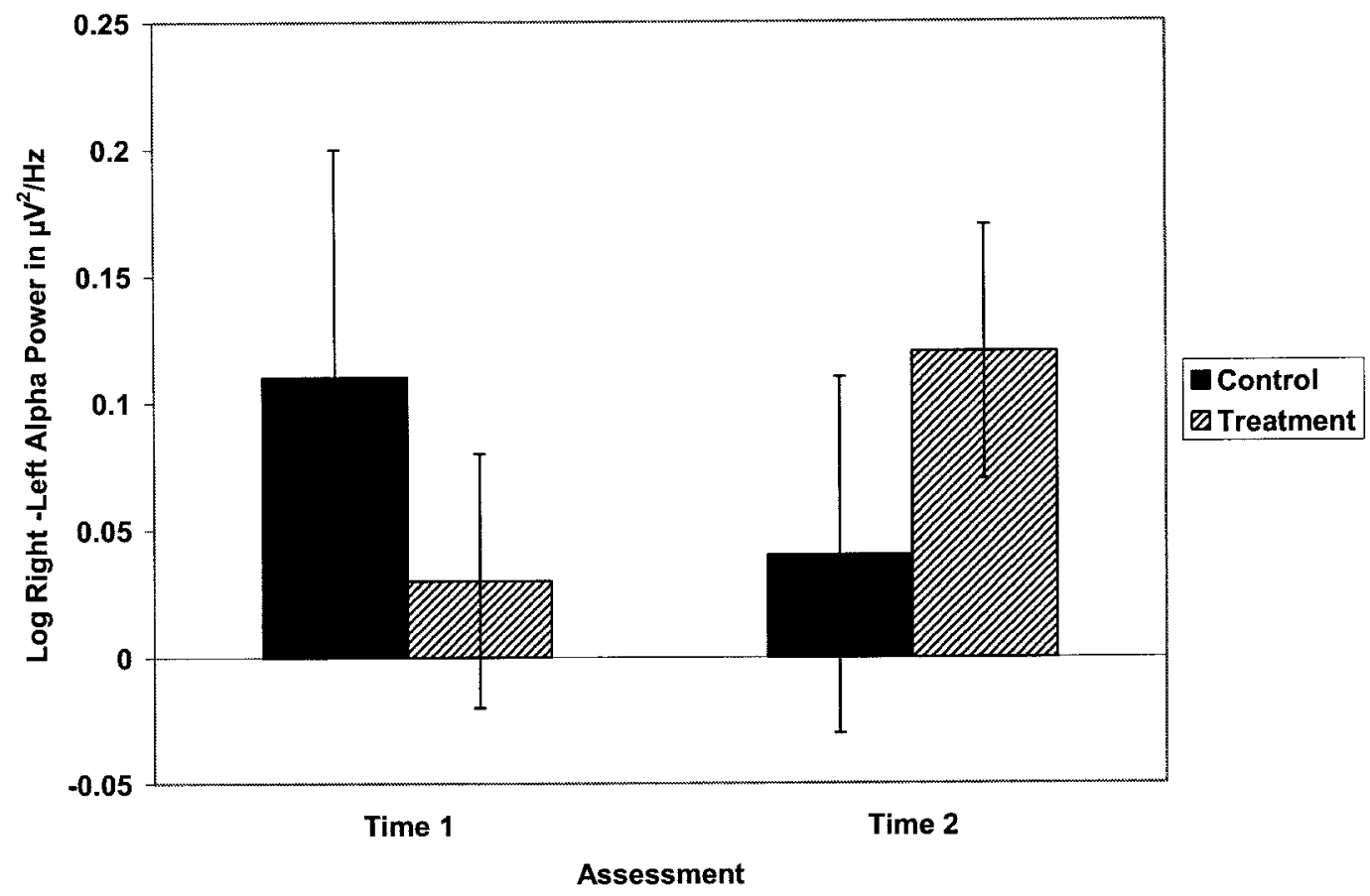

Fig. 3. Means \pm SE asymmetric activation (in the T3/T4 electrode sites) in response to the positive emotion induction in the Meditation group and Control group during Times 1 and 2. The ordinate is the same metric of asymmetric activation displayed in Figure 2.

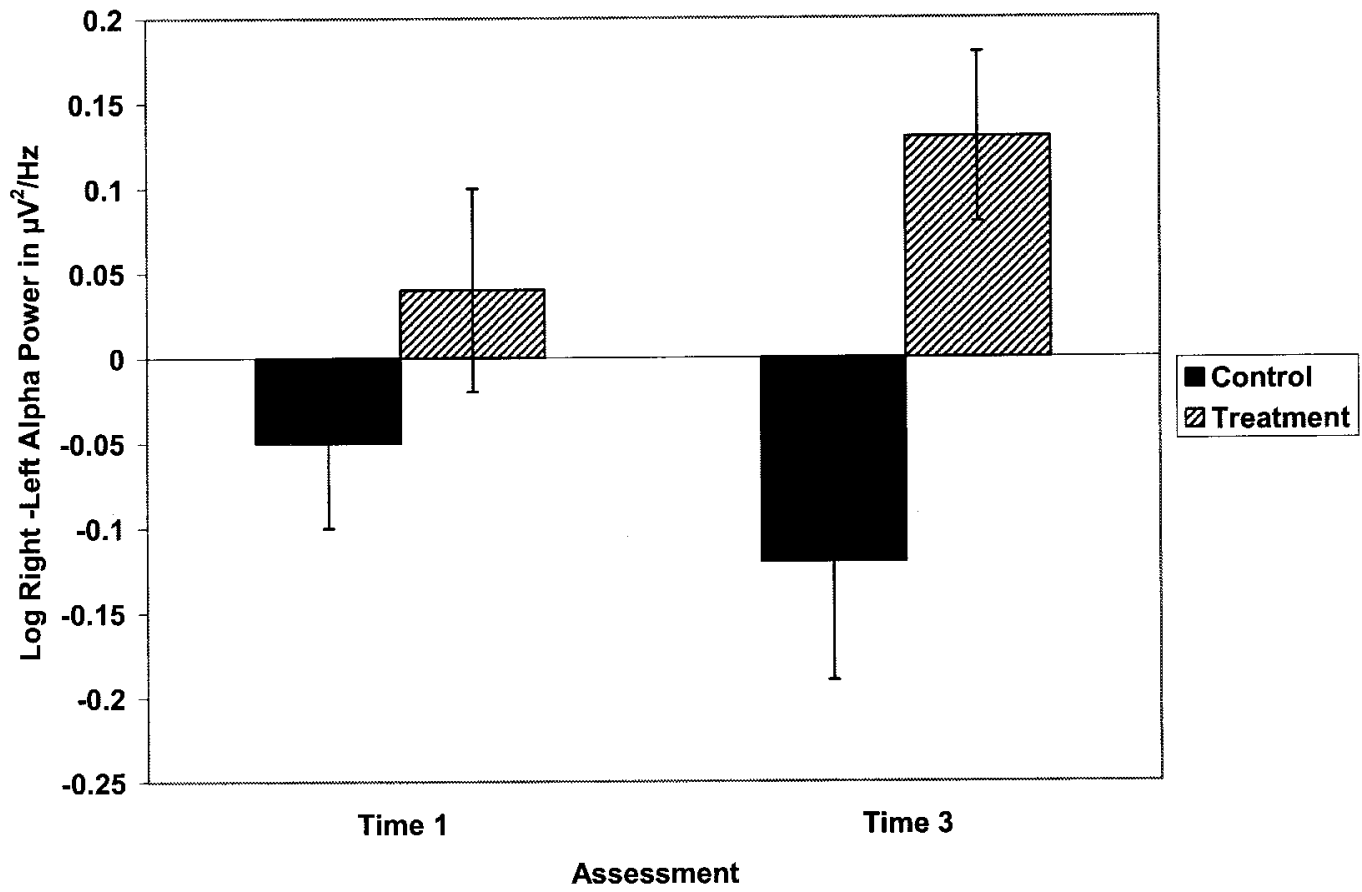

Fig. 4. Means \pm SE asymmetric activation in response to the negative emotion induction in the Meditation group and Control group during Times 1 and 3 . The ordinate is the same metric of asymmetric activation displayed in Figure 2 (C3/C4).

\section{Relations Among Measures}

To examine the relation between the magnitude of increase in left anterior activation and the magnitude of antibody titer rise in response to the influenza vaccine from the 4- to 8-week blood draw, we computed a change score for each subject to express the change in activation asymmetry from Time 1 to Times 2 and 3 and correlated the change in activation asymmetry with the rise in antibody titers, separately for each group. Among subjects in the meditation group, those who showed a greater increase in left-sided activation from Time 1 


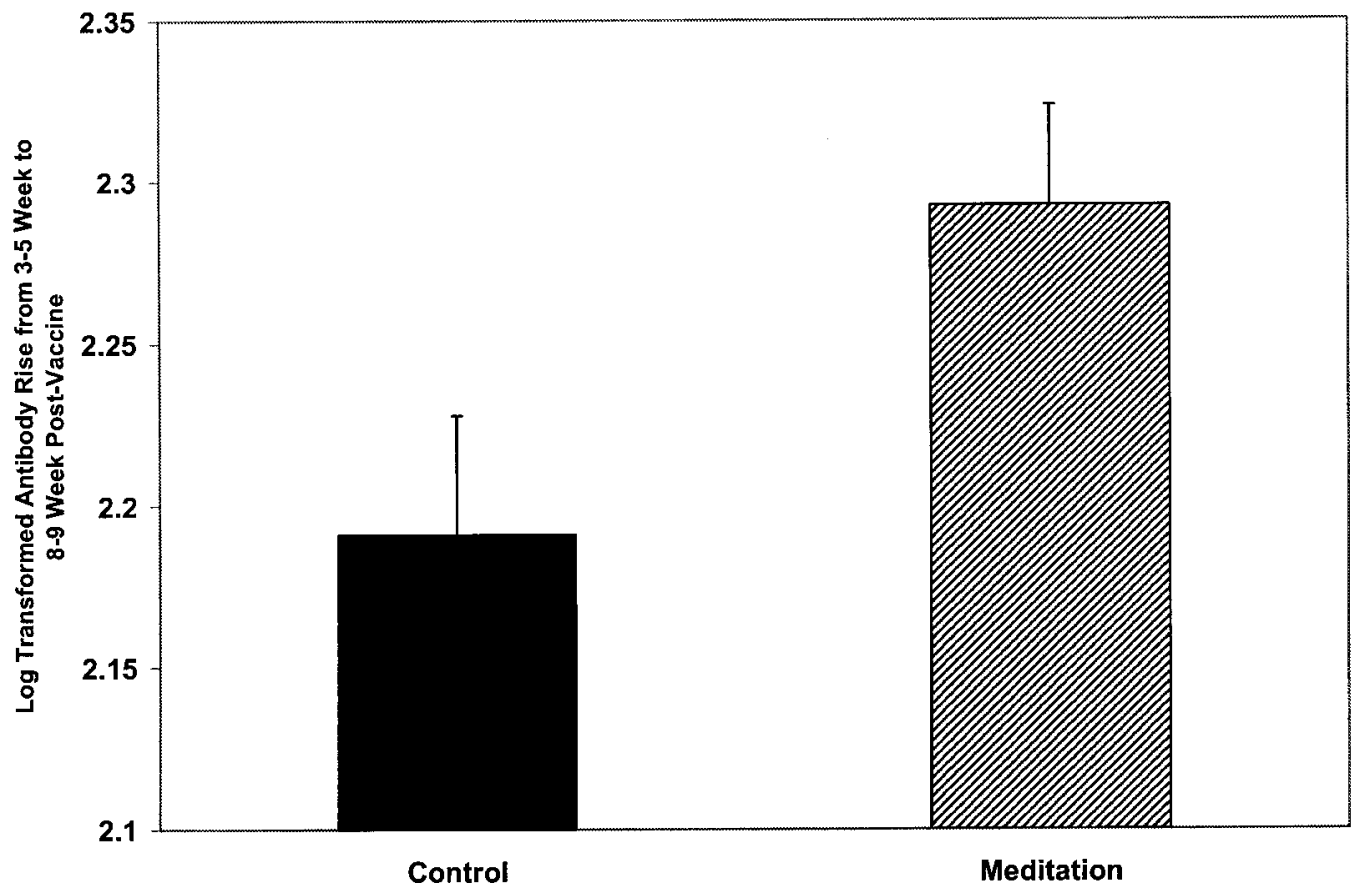

Fig. 5. Means \pm SE antibody rise from the 3- to 5-week to the 8- to 9-week blood draw in the Meditation and Control groups. The ordinate displays the difference in the log-transformed antibody rise between the 3- to 5- and the 8- to 9-week blood draws derived from the hemagglutination inhibition assay.

to Time 2 displayed a larger rise in antibody titers $(r=.53, p$ $<.05$; see Figure 6 ) while there was no significant relation between these variables for subjects in the control group $(r=$ .26). These correlations were not significantly different.

We also examined correlations between the frequency and duration of reported practice and changes in the self-report and EEG measures that showed significant Group $\times$ Time interactions, as well as antibody titers to influenza vaccine. There were no significant associations between the measures of practice and any of the biological or self-report measures. Descriptive statistics on these measures of daily practice are provided in Table 1.

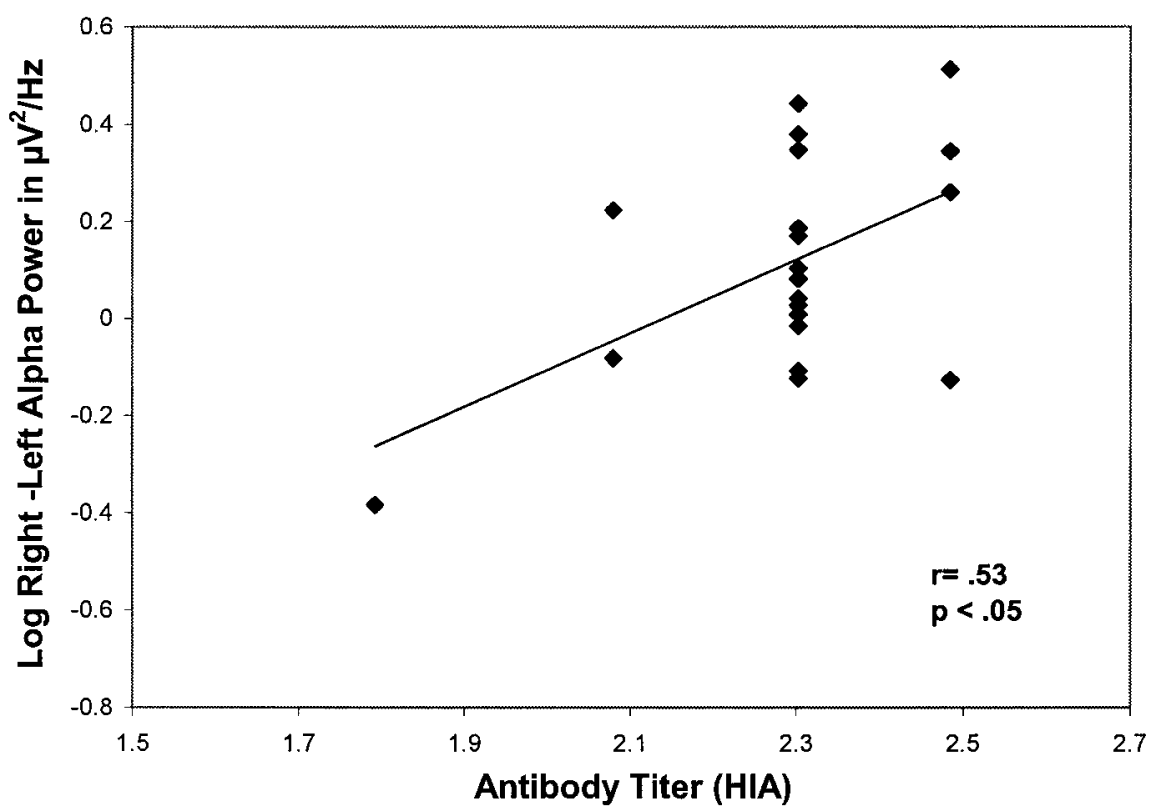

Fig. 6. Scatter plot for the meditation group only showing the relation between the change in asymmetric anterior activation at baseline from Time 1 to Time 2 in $\mathrm{C} 3 / \mathrm{C} 4$ and the magnitude of rise in antibody titers to the influenza vaccine from the week 3 to 5 to the week 8 to 9 blood draw. The meditators that showed the largest magnitude increase in left-sided anterior activation from Time 1 to Time 2 also showed the largest rise in antibody titers from the 3- to 5- to 8- to 9-week blood draws. There was no significant relation between these variables in the control group. 
TABLE 1. Self-Reported Daily Practice in the Meditation Group

\begin{tabular}{|c|c|c|c|c|c|c|}
\hline & \multicolumn{3}{|c|}{ Time 2} & \multicolumn{3}{|c|}{ Time 3} \\
\hline & Means & SD & Range & Means & SD & Range \\
\hline How regularly do you practice (0-6 scale) & 2.48 & 2.14 & $0-6$ & 1.70 & 1.66 & $0-6$ \\
\hline How long do you practice (0-30 minutes/session) & $16.19 \min$ & 9.74 & $0-30$ & $14.21 \mathrm{~min}$ & 13.36 & $0-30$ \\
\hline How many times have you practiced in the last week? & 2.52 & 2.56 & $0-7$ & 2.15 & 2.03 & $0-7$ \\
\hline
\end{tabular}

\section{DISCUSSION}

These findings are the first to document significant changes in anterior activation asymmetry as a function of meditation training. A variety of previous research has established that activation asymmetries in anterior scalp regions are related to dispositional affect. Moreover, such asymmetries reflect both state and trait components $(32,33)$ with both phasic positive mood as well as dispositional positive affect associated with greater relative left-sided anterior activation. On the basis of an extensive corpus of both animal and human data, Davidson and colleagues recently suggested (33) that prefrontal activation asymmetries are plastic and could be shaped by training. The findings from this study are the first to suggest that meditation can produce increases in relative left-sided anterior activation that are associated with reductions in anxiety and negative affect and increases in positive affect.

We predicted that we would find significant changes in prefrontal as well as central electrode locations. It is unclear why our most consistent findings were observed at the central leads $(\mathrm{C} 3 / \mathrm{C} 4)$, although this is a region where we have observed reliable affect-related asymmetries in the past (11). Moreover, we have found robust asymmetric increases in left premotor activation in response to positive emotional pictures in a study that measured regional glucose metabolism with positron emission tomography (34). The fact that there was no significant increase in dispositional positive affect in the meditation group may be related to the failure to detect increases in left prefrontal activation. It may well be that if the duration and/or intensity of the intervention were increased, the increases would be observed in both positive affect and left prefrontal activation.

It is of interest that we observed reliable increases in left-sided activation with training in the meditation group in response to both the positive and negative affect induction. We have suggested on the basis of a growing literature on the neural bases of emotion regulation that left-sided anterior activation is associated with more adaptive responding to negative and/or stressful events. Specifically, individuals with greater left-sided anterior activation have been found to show faster recovery after a negative provocation (see Refs. 32 and 33 for reviews).

To our knowledge, this is the first demonstration of a reliable effect of meditation on an in vivo measure of immune function. The finding may reflect a relatively more rapid peak rise in antibody titers among the meditators compared with the controls. The observation that the magnitude of change in immune function was greater for those subjects showing the larger shift toward left-sided activation further supports earlier associations between these indices $(12,13)$.

There are several limitations of our study that are important to note. First, there was a relatively small number of subjects who participated and this limited our statistical power. A number of our hypothesized effects were in the predicted direction, but failed to reach significance. Second, the study examined the impact of a relatively brief intervention delivered in a demanding work environment during regular business hours. It will be of interest in the future to examine the changes in brain and immune function produced by MBSR or more intensive training in a more conducive learning environment. And, finally, the measures of brain function we obtained are relatively crude (see Ref. 20 for a discussion of their limitations). Future studies should examine the impact of meditation using more neuroanatomically informative measures of brain function such as functional magnetic resonance imaging.

Our findings indicate that a short training program in mindfulness meditation (MBSR) has demonstrable effects on brain and immune function and underscores the need for additional research on the biological consequences of this intervention.

The authors thank the John D. and Catherine T. MacArthur Foundation's Research Network on Mind-Body interaction, The Fetzer Institute, and National Institutes for Mental Health (Grant P50 MH61083), and Anne Skillings for technical support during the intervention phase of the study.

\section{REFERENCES}

1. Lou HC, Kjaer TW, Friberg L, Wildschiodtz G, Holm S, Nowak M. A 150-H2O PET study of meditation and the resting state of normal consciousness. Hum Brain Map 1999;7:98-105.

2. Jevning R, Anand R, Biedebach M, Fernando G. Effects on regional cerebral blood flow of transcendental meditation. Physiol Behav 1996; 59:399-402.

3. Herzog H, Lele VR, Kuwert T, Langen KJ, Kops ER, Feinendegen LE. Changed pattern of regional glucose metabolism during yoga meditative relaxation. Neuropsychobiology 1990;23:182-7.

4. Kabat-Zinn J, Massion AO, Kristeller J, Peterson LG, Fletcher KE, Pbert L, Lenderking WR, Santorelli SF. Effectiveness of a meditation-based stress reduction program in the treatment of anxiety disorders. Am J Psych 1992;149:936-43.

5. Miller JJ, Fletcher K, Kabat-Zinn J. Three-year follow-up and clinical implications of a mindfulness meditation-based stress reduction intervention in the treatment of anxiety disorders. Gen Hosp Psych 1995;17: 192-200.

6. Teasdale JD, Segal ZV, Williams JM, Ridgeway VA, Soulsby JM, Lau MA. Prevention of relapse/recurrence in major depression by mindfulness-based cognitive therapy. J Consult Clin Psychol 2000;68:615-23.

7. Teasdale JD, Segal Z, Williams MG. How does cognitive therapy prevent 


\section{R. J. DAVIDSON et al.}

depressive relapse and why should attentional control (mindfulness) training help? Behav Res Ther 1995;33:25-39.

8. Beauchamp-Turner DL, Levinson DM. Effects of meditation on stress, health, and affect. Medical-Psychother: Int J 1992;5:123-31.

9. Davidson RJ, Irwin W. The functional neuroanatomy of emotion and affective style. Trends Cogn Sci 1999;3:11-21.

10. Davidson RJ. Emotion and affective style: hemispheric substrates. Psychol Sci 1992;3:39-43.

11. Davidson RJ, Ekman P, Saron C, Senulis J, Friesen WV. Approach/ withdrawal and cerebral asymmetry: Emotional expression and brain physiology, I. J Pers Soc Psychol 1990;58:330-41.

12. Kang DH, Davidson RJ, Coe CL, Wheeler RW, Tomarken AJ, Ershler WB. Frontal brain asymmetry and immune function. Behav Neurosci 1991;105:860-9.

13. Davidson RJ, Coe CC, Dolski I, Donzella B. Individual differences in prefrontal activation asymmetry predict natural killer cell activity at rest and in response to challenge. Brain Behav Immun 1999;13:93-108.

14. Solberg EE, Halvorsen R, Sundgot-Borgen J, Ingjer F, Holen A. Meditation: a modulator of the immune response to physical stress? A brief report. Br J Sports Med 1995;29:255-7.

15. Kiecolt-Glaser JK, Garner W, Speicher CE, Penn GM, Holiday J, Glaser R. Psychosocial modifiers of immunocompetence in medical students. Psychosom Med 1984;46:7-14.

16. Glaser R, Kiecolt-Glaser JK, Malarkey WB, Sheridan JF. The influence of psychological stress on the immune response to vaccines. Ann NY Acad Sci 1998;47:113-142.

17. Cohen S, Herbert TB. Health psychology: Psychological factors and physical disease from the perspective of human psychoneuroimmunology. Ann Rev Psychol 1996;47:113-42.

18. Antoni MH. Cognitive-based stress management intervention effects on anxiety, 24-hr urinary norepinephrine output, and T-cytotoxic/suppressor cells over time among symptomatic HIV infected gay men. J Consult Clin Psychol 2000;68:31-45.

19. Kiecolt-Glaser JK, Glaser R, Gravenstein S, Malarkey WB, Sheridan J. Chronic stress alters the immune response to influenza virus vaccine in older adults. Proc Natl Acad Sci USA 1996;93:3043-7.

20. Davidson RJ, Jackson DC, Larson CL. Human electroencephalography. In: Cacioppo JT, Bernston GG, Tassinary LG, editors. Principles of psychophysiology. 2nd ed. New York: Cambridge University Press; 2000. p. $27-52$
21. Pivik T, Broughton R, Coppola R, Davidson RJ, Fox NA, Nuwer R. Guidelines for quantitative electroencephalography in research contexts. Psychophys 1993;30:547-58.

22. Tomarken AJ, Davidson RJ, Wheeler RE, Kinney L. Psychometric properties of resting anterior EEG asymmetry: temporal stability and internal consistency. Psychophys 1992;29:576-92.

23. Watson D, Clark LA, Tellegen A. Developmental and validation of brief measures of positive and negative affect: The PANAS scales. J Pers Soc Psych 1988;54:1063-70.

24. Spielberger CD, Gorsuch RL, Lushene RE, Vagg PR, Jacobs GA. Manual for the state-trait anxiety inventory. Palo Alto CA: Consulting Psychologists; 1983.

25. Kabat-Zinn J. Full catastrophe living: using the wisdom of your body and mind to face stress, pain and illness. New York: Delacorte; 1990.

26. Santorelli S. Heal thy self: lessons on mindfulness in medicine. New York: Random House; 1999.

27. Kabat-Zinn J. Mindfulness-based stress reduction: Past, present, and future. Clin Psychol Sci Pract, 2003. In press.

28. Shapiro SL, Schwartz GE, Bonner G. Effects of mindfulness-based stress reduction on medical and premedical students. J Behav Med 1998;21: 581-599.

29. Kabat-Zinn J, Wheeler E, Light T, Skillings A, Scharf M, Cropley TG, Hosmer D, Bernhard J. Influence of a mindfulness-based stress reduction intervention on rates of skin clearing in patients with moderate to severe psoriasis undergoing phototherapy (UVB) and photochemotherapy (PUVA). Psychom Med 1998;60:625-632.

30. Bishop SR. What do we really know about mindfulness-based stress reduction? Psychom Med 2002; 64:71-84.

31. Baer R. Mindfulness training as a clinical intervention: A conceptual and clinical review. Clin Psychol Sci Pract 2003. In press.

32. Davidson RJ. Affective style, psychopathology, and resilience: brain mechanisms and plasticity. Am Psychol 2000;55:1196-1214

33. Davidson RJ, Jackson DC, Kalin NH. Emotion, plasticity, context, and regulation: perspectives from affective neuroscience. Psychol Bull 2000; 126:890-909.

34. Sutton SK, Larson CL, Ward RT, Holden JE, Perlman SB, Davidson RJ. The functional neuroanatomy of the appetitive and aversive motivation systems: Results from an FDG-PET study. Neuroimage 1996;3:S240. 